\title{
The Time-Dependent Dynamics of Gut Microbiota and the Interaction With Host Metabolism in Mouse Pulmonary Fibrosis Models
}

\section{Xiao-Hui Yang}

Affiliated Hospital of Qingdao University

Xiu-Juan Jia

Affiliated Hospital of Qingdao University

\section{Xiao-Sa Chi}

Affiliated Hospital of Qingdao University

\section{Xiao-Meng Wang}

Affiliated Hospital of Qingdao University

\section{Fang-Fang Wang}

Affiliated Hospital of Qingdao University

\section{Jin-Peng Cong}

Affiliated Hospital of Qingdao University

Hong-Yun Wei

Affiliated Hospital of Qingdao University

Hui Zheng

Wuhan Metware Biotechnology Co., Ltd

Yu-Zhu Zhang ( $\square$ zhangyz0901@163.com )

Affiliated Hospital of Qingdao University

\section{Research Article}

Keywords: Pulmonary fibrosis, gut microbiota, serum metabolites, metagenome, metabolic disorders, mice

Posted Date: September 30th, 2021

DOI: https://doi.org/10.21203/rs.3.rs-882650/v1

License: (c) (1) This work is licensed under a Creative Commons Attribution 4.0 International License.

Read Full License 
The Time-dependent dynamics of gut microbiota and the interaction with host metabolism in mouse pulmonary fibrosis models

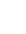

Xiao-Hui Yang ${ }^{1}$, Xiu-Juan $\mathrm{Jia}^{2}$, Xiao-Sa Chi ${ }^{2}$, Xiao-Meng Wang ${ }^{2}$, Fang-Fang Wang ${ }^{1}$, Jin-Peng Cong ${ }^{1}$, Hong-Yun $\mathrm{Wei}^{3}$, Hui Zheng ${ }^{4}$, Yu-Zhu Zhang ${ }^{2 *}$

${ }^{1}$ Department of Respiratory and Critical Care Medicine, Affiliated Hospital of Qingdao University, Qingdao 266000, China

${ }^{2}$ Department of Geriatrics, Affiliated Hospital of Qingdao University, Qingdao 266000, China

${ }^{3}$ Department of Gastroenterology, Affiliated Hospital of Qingdao University, Qingdao 266000, China

${ }^{4}$ Wuhan Metware Biotechnology Co., Ltd., Wuhan 430000, China

\section{*Correspondence:}

Yu-Zhu Zhang,

Department of Geriatrics,

Affiliated Hospital of Qingdao University,

Qingdao 266000, P. R. China

Email: zhangyz0901@,163.com 


\section{Abstract}

Background: Pulmonary fibrosis (PF) is a chronic progressive disease whose pathogenesis is thought to be associated with activation of the immune system and consequent metabolic changes. Recent studies suggested that gut microbes are closely related with host's immune response and metabolic changes in fibrotic hosts. However, the dynamic changes of the gut microbiome and the interaction profiles with host metabolism during the development of pulmonary fibrosis remain inconclusive.

Results: We collected serum and fecal samples from bleomycin-induced fibrotic mice at $0,7,14$, and 28 days and performed UPLC-MS analysis on serum metabolites and metagenomic sequencing on fecal samples. It is found that the serum metabolic profile and gut microbiome were significantly altered in mice during the progression of fibrosis. Among the serum metabolites, the levels of three major types of lipids, i.e., glycerolipids, glycerophospholipids, and fatty acids exhibit significant time-dependent changes.

The glycerolipid TG and multiple glycerophospholipids (3 PG, 6 PE, and 1 PC) decreased in the early stage of fibrosis and increased in the late stage. The other two types of glycerolipids MG and DG and the fatty acids Cartinine and Punicic acid decreased through the development of fibrosis. In the meantime, we detected significantly elevated abundance of gut microbiome taxa, including Prevotella sp. from Bacteroidetes, Lactobacillus from Firmicutes, and Bifidobacterium from Actinobacteria in mice with pulmonary fibrosis. When compared to the dynamic profiles of serum metabolites, the abundances of gut microbiome show a high level of 
correlation with that of serum metabolites. The taxa from Bacteroides, such as Butyricimonas_synergistica and Muribaculaceae, show positive correlation with the cluster of glycerophospholipids, while taxa from Firmicutes, such as Clostridioides difficile and Enterococcus faecium exhibit negative correlation. Further functional classification suggested that those taxa are involved in multiple functional modules, such as Transporters, Secretion system, and Metabolism.

Conclusions: The results reveal the synergistic changes between the gut microbiome and host metabolism and the dynamic responses of gut microbiome to host fibrosis during the progression of fibrosis.

Keywords: Pulmonary fibrosis, gut microbiota, serum metabolites, metagenome, metabolic disorders, mice

\section{INTRODUCTION}

Pulmonary fibrosis (PF) is a chronic progressive disease with excessive deposition of collagen tissue in the lung interstitium. It is a common and irreversible interstitial lung disease and one of the serious diseases of the respiratory system [1]. The patients would develop lung fibroblast proliferation, extracellular matrix collagen deposition, airway inflammatory cell infiltration, etc., which eventually led to alveolar and lung tissue fibrosis [2]. Due to the lack of reliable early diagnosis methods and effective treatment methods to reverse the natural process and outcome of fibrosis, the median survival time from diagnosis was only 2.5-3.5 years, and the 5-year survival rate was less than $40 \%[3,4]$. Pulmonary fibrosis may be even more malignant than some malignant tumors because of progressive respiratory failure. 
Pulmonary fibrosis is usually caused by different stimuli such as toxin, infection, severe trauma, autoimmune reaction, adverse drug reaction, and idiopathic, unknown factors $[5,6]$. The pathogenesis of pulmonary fibrosis is not completely clear. At present, it is believed that the development of pulmonary fibrosis can be divided into two stages, namely the early stage of alveolar inflammation and the late stage of excessive fibrotic repair. In the early stage of alveolar inflammation, the pulmonary activated interstitial and infiltrating cells will secrete a series of cytokines, including tumor necrosis factor (TNF-a), chemokines (CXC), and adhesion cytokines (CAM), which affect each other and promote further aggregation of inflammatory monocytes; in the later stage of fibrosis, inflammatory cells and interstitial cells (fibroblasts and myofibroblasts) were activated to secrete factors such as growth factor, interleukin (IL) and matrix metalloproteinase (MMPs), which were involved in the repair and remodeling of lung tissue and blood vessels, and eventually led to the occurrence of pulmonary fibrosis [7-9].

In the process of pulmonary fibrosis, a series of metabolite changes occurred. Pulmonary fibrosis can lead to glycolysis reprogramming. Inhibition of mouse glycolytic enzyme 6-fructose phosphate-2-kinase/fructose-2 by pharmacological or genetic methods, 6-Diphosphatease 3 (PFKFB3) not only reduced fibroblast activation in vitro but also significantly improved the severity of pulmonary fibrosis [10]. Subsequent studies confirmed that this metabolic reprogramming was closely related to macrophage activation, and the M2 macrophage distribution of fibrosis in fibrotic alveolar was independent of fatty acid oxidation and synthesis or lipolysis but 
relied on glycolytic [11].

With the development of pulmonary fibrosis, the metabolome of the organism is also altered. Sung-Woo Park et al. analyzed lung tissue using mass spectrometry and identified 25 altered metabolites associated with pulmonary fibrosis, mainly involved in metabolic pathways such as the adenosine triphosphate degradation pathway and the glycolytic pathway [12]. Cédric Bovet et al. tested serum from patients with pulmonary fibrosis, finding that LysoPC has the potential of serving as a biomarker for pulmonary fibrosis [13].

A variety of evidences show that some diseases can cause changes in gut microbiota, and meanwhile, gut microbiota plays a role in immune protection against lung diseases (such as pneumonia and asthma) [14, 15]. Marie-Clair Arrieta et al. analyzed the metabolites of the feces and urine of infant mice of different ages, and demonstrated that the composition and abundance of the gut microbiota at the age of 3 months affect the risk of human asthma [16]. Its main research methods included 16S rRNA gene sequencing and whole-genome sequencing. Jin $\mathrm{Su}$ et al. studied the feces of mice with pulmonary fibrosis using metabolomic and 16S rDNA sequencing techniques, revealing a previously unknown link between the intestine and pulmonary fibrosis [17]. Although adverse changes in the intestinal ecosystem have been identified in multiple diseases, such as asthma, lung cancer, and acute lung injury, the details about the intestinal microbiome and the interaction between the microbiome and host metabolism in pulmonary fibrosis models have not yet been fully elucidated. $[16,18-21]$ 
In the present study, we aimed to examine the interactions between the gut microbiota and serum metabolome, and investigate their dynamic changes in mice with pulmonary fibrosis using multi-omics approaches.

\section{MATERIALS AND METHODS}

\section{Animal, Fibrotic Models and Sample Collection}

All experiments were approved by the Ethics Committee of Affiliated Hospital of Qingdao University (Ethics NO. QYFYW2LL26275). Specific-pathogen-free C57BL/6 J male mice (about 8 weeks of age) were purchased from Beijing Vital River Laboratory Animal Technology Co., Ltd.

All mice were housed in a barrier facility and were fed normal-chow diet. They were randomly assigned to the four groups (control, model_7Day, model_14Day, model_28days) with 10 mice in each group. To induce pulmonary fibrosis, mice were injected intraperitoneally and anesthetized suitably with $1 \%$ pentobarbital sodium, and then vertically mounted on a slope. A laryngoscope was held at the throat of the mice and the tongue was pulled lightly, a hermetic venous indwelling needle was plugged in and the inner tube was taken out, the drug $(2.0 \mathrm{mg} / \mathrm{kg}$ bleomycin for experimental group, the same amount of normal saline for normal group) was dropped into the trachea, the tube was unplugged and the mice laid in a mouse cage.

Lung tissue, serum, and fecal samples were collected for histopathological, metabolomic, and metagenomic assays from drug-induced mice at 7, 14, and 28 days as well as from control mice. 
Mice were deeply anesthetized with sodium pentobarbital and died from blood

135 loss after incision of the abdominal aorta. After induction of pulmonary fibrosis, the

136 heart was perfused with PBS and the left lobe was injected with approximately $250 \mu 1$

$1374 \%$ paraformaldehyde, and then the 4\% paraformaldehyde-fixed tissue was routinely

138 processed, embedded in paraffin, and sectioned. Two adjacent slices were stained with

139 TGF- $\beta$, HE, and Masson, respectively, and the stained slides were scanned with

140 PANNORAMIC DESK/MIDI/250/1000 (3DHISTECH, Hungary), and representative

141 slice areas are shown in the screenshots.

142 UPLC-MS Metabolome Profiling

143 Metabolite extracts were obtained from serum samples following

144 methanol-assisted protein precipitation and then analyzed using an LC-ESI-MS/MS

145 system (UPLC, ExionLC AD, https://sciex.com.cn/; MS, QTRAP® System,

146 https://sciex.com/). Liquid phase separation was performed using a Waters ACQUITY

147 UPLC HSS T3 C18 column $(1.8 \mu \mathrm{m}, 2.1 \mathrm{~mm} * 100 \mathrm{~mm})$. LIT and triple quadrupole

148 (QQQ) scans were acquired on a triple quadrupole linear ion trap mass spectrometer

149 (QTRAP) controlled by Analyst 1.6.3 software (Sciex) QTRAP® LC-MS/MS system.

150 A specific set of MRM shifts was monitored for each period based on the metabolite

151 elution in each period. Software Analyst 1.6.3 was used to process the mass

152 spectrometry data. Several existing mass spectrometry public databases were

153 consulted for metabolite structure analysis, mainly massbank

154 (http://www.massbank.jp/), knapsack (http://kanaya.naist.jp/knapsack/), HMDB 
(http://www.hmdb.ca/) and Metlin (http://metlin.scripps.edu/index.php). Qualitative

analysis was performed based on retention times and mass-to-charge ratios of parent and daughter ions of test substances from internal and other public databases. The quantitative analysis was performed by the triple quadrupole mass spectrometry multiple reaction monitoring (MRM) method. The signal intensity of the characteristic ions is obtained in the detector. MultiQuant is used to integrate and calibrate the chromatographic peaks. The peak area of each chromatographic peak represents the relative amount of the corresponding substance[22, 23].

The Kruskal-Wallis H test was used to determine the differences between groups. The lipidomics data were normalized using R software (https://www.r-project.org/). SIMCA 14.1 was used to analyze the metabolites using principal component analysis (PCA) and orthogonal partial least squares discrimination analysis (OPLS-DA) model. VIP $>1$ and $\mathrm{P}$ values $<0.05$ were considered significant. Asterisks represent: ns means no significant, $* \mathrm{P}<0.05, * * \mathrm{P}<0.01, * * * \mathrm{P}<0.001$ and $* * * * \mathrm{P}<0.0001$.

\section{DNA Isolation and Metagenomic Sequencing and Assembly}

Total genomic DNA was extracted from fecal samples using the Mag-bind Soil DNA Kit (Omega Biotek, USA) according to the manufacturer's instructions. Approximately $2 \mu \mathrm{g}$ of DNA was prepared per sample. Sequence libraries were generated using NEBNext ${ }^{\circledR}$ Ultra $^{\mathrm{TM}}$ DNA Library Prep Kit for Illumina (NEB, USA). The libraries were sequenced on the Illumina NovaSeq /HiSeq platform (insert size $350 \mathrm{bp}$, read length $150 \mathrm{bp}$ ) at the Novogene Bioinformatics Technology Co., Ltd. (Tianjin, China). FastQC is used to assess the quality of raw data comprehensively 
177 generated by sequencing, while we screened the data for quality, removing sequences

178 less than $50 \mathrm{bp}$ in length or containing ambiguous bases. All metagenomic raw data

179 have been submitted to the NCBI database (accession: PRJNA722397).

180 Gene Catalog (Unigenes) Construct and Taxonomy Predication Prediction of genes using MetaGeneMark version 2.10

(http://exon.gatech.edu/GeneMark/) $\quad$ [24]. Redundant genes (95\% identity, $90 \%$ overlap) were removed by MMseqs2, resulting in a non-redundant gene catalog. Taxonomic function assignment and taxonomic identification of the readings using MEGAHIT. The LCA algorithm of the MEGAN software system was used for annotation.

\section{Gene Functional Annotations}

Predicted unigenes were assigned to the Kyoto Encyclopedia of Genes and Genomes (KEGG) using KOBAS, the Carbohydrate-Active Enzyme Database (CAZy), and the Gene Evolutionary Lineage: Unsupervised Orthologous Group (eggNOG) using Diamond and the Carbohydrate-Active Enzyme Database (CAZy) using eggnog-mapper. The abundance of each functional annotation is the sum of the abundance of each functional level annotation.

\section{Correlation Analysis of Gut Microbiota and Metabolites}

To determine the association between gut microbiota and metabolites in the pulmonary fibrosis model, we constructed a correlation analysis between gut 
3.4.3 (Hmisc package). The first 52 species and metabolites between the four groups were analyzed.

\section{Data Analysis}

To identify features (taxa and functional modules) differentially represented between the four groups, differential abundance taxa or functional modules were selected using LEfSe (linear discriminant analysis (LDA)) effect sizes. Differential abundance of phylum, genus, species and functional modules between any two groups was tested by metagenomeSeq [25], and P values were corrected for false discovery rate (FDR) using the Benjamini-Hochberg method.

\section{RESULTS}

\section{The Establishment of Pulmonary Fibrosis Mouse Models Induced by Bleomycin}

We used bleomycin-induced mice to construct a pulmonary fibrosis model and collected their lung tissues, serum, and feces at four time points, i.e., 0 day (control), 7th day (Model_7Day), 14th day (Model_14Day), and 28th day (Model_28Day) (Figure 1A). No significant changes in body weight and dietary intake were observed in bleomycin-induced mice as compared to the control group ( $p>0.05)$. TGF- $\beta$ western blot and immunohistochemistry of mice lung tissues showed that there exist typical and large fibrotic lesions in the models (Figure 1B and Figure 1D), although TGF- $\beta$ secretion of lung tissues decreased in 28 days. Immunohistochemistry on lung tissue sections in mice further demonstrated that bleomycin induced strong fibrosis in the lungs of mice (Figure 1C). Together, the above data demonstrated that the serum 
and stool samples in the model were reliable for further gut microbiota sequencing

221 and metabolomic assays.

\section{The Altered Metabolism in Serum of Mouse Fibrosis Models}

The time-series metabolic profile in the serum of the mouse fibrosis models was obtained using mass spectrometry (MS) assays. Clustering analysis based on PCA and OPLS-DA showed clear separation between the control and fibrosis models, indicating the metabolic shift in the fibrosis models as compared to the control (Figure 2A). A total of 585, 626, 141 unique metabolites are differentially represented in the Model_7Day, Model_14Day, Model_28days, respectively in comparison with the control (VIP $>1$ and p-value $<0.05)$ (Table S1). 49 top metabolites significantly changed in all three fibrotic models are presented in the hierarchical clustering heatmap (Figure 2B and Table S2). It is shown that those metabolites form four clusters (C1-C4) and are dominated by three types of lipids, i.e., glycerophospholipids, glycerolipids, and fatty acids. Glycerophospholipids constitute the majority of the cluster C2 (including 3 PG, 6 PE, and 1 PC) and exhibit a time-dependent pattern of decreasing in the early stage of fibrosis (Model_7Day and Model_14Day) and increasing in the late stage (Model_28Day), while both glycerolipids and fatty acids fall into multiple clusters with divergent time-dependent patterns. For example, the tri-radyl type of glycerolipid TG clusters together with glycerophospholipids in C2 by sharing similar time-dependent pattern, whereas the duo-radyl and mono-radyl type of glycerolipid (MG and DG) falls into the cluster $\mathrm{C} 4$, where the abundances of the metabolites decreased through the development of fibrosis. The fatty acids are 
scattered in the cluster C4 (such as Cartinine and Punicic acid) and C1 (such as

243 Linoleic acid, Hexadecanoic acid, Elaidic acid, and 8,11-icosadienoic acid). In

244 addition, the nucleotide Adenosine is special in that its abundance is only increased in

245 the Model_14Day, distinguished from other metabolites.

Further pathway enrichment analysis identified five pathways

247 (glycerophospholipid metabolism, glycerolipid metabolism, linoleic acid metabolism,

248 sphingolipid metabolism, and Arginine biosynthesis) enriched with the differentially

249 represented metabolites across the three time points, indicating the relationship

250 between pulmonary fibrosis and aberrant metabolism of the major lipids in serum,

251 such as glycerophospholipid, glycerolipid, fatty acids, and sphingolipid (Figure

252 2C-2D).

253

254

255

256

257

258

259

260

\section{Alterations in Gut Microbial Compositions in the Mouse Fibrosis Models}

To evaluate the changes of intestinal microbes in the process of lung fiber formation in mice, we performed metagenomic testing on the feces of mice. The optimized reads ranging from $74,230,682$ to $99,459,116$ were obtained from all samples. The average quality distribution of sequences showed a peak width of 36 for the quality Q-value distribution of sequencing data, indicating high sequencing quality. After data quality control, a high percentage of valid sequences ( $99 \%)$ was achieved in all samples. This indicates that the sequencing depth was sufficient for the investigation of the fecal microbiota.

The taxonomic abundance analysis showed that the gut microbiota of the mouse models was dominated by families from Firmicutes, Bacteroidetes, Proteobacteria, 
and Actinobacteria. There are no significant differences in the taxonomic diversity among the four groups based on the alpha diversity calculation (Shannon, Kruskal-Wallis test, $p=0.2075$ ) (Figure 3A). Principal coordinates analysis (PCoA) showed that the Model_7Day and Model_14Day are closer to each other, while the control and Model_28Day gathered together, with the first (PC1) and second (PC2) principal coordinate accounting for $19.9 \%$ and $14.9 \%$ of the total variation, respectively (Figure 3B). In order to identify the discriminating taxa in each of the four mouse groups, we applied LEfSe (LDA Effect Size) analysis to the samples and identified 79 taxa with differential abundance across the four groups (Table S3). Their discriminating ability indicated as LDA (linear discriminant analysis) score in Figure $3 \mathrm{C}$ revealed that the control group is characterized by a high abundance of Verrucomicrobiae (such as Akkermansia muciniphila), the Model_7Day is enriched with phylotypes from Bacteroidetes (such as Prevotella sp.) and Proteobacteria (such as Pandoraea sputorum), the Model_14Day is enriched with phylotypes from Firmicutes (such as Lactobacillus), while the Model_28Day contains abundant species from Actinobacteria, Bacteroidetes and Proteobacteria. It is seen that although the overall diversity is no significantly different between the four groups of mice, the abundance of specific taxa has altered during the progression of fibrosis. Notably, several phylotypes from Verrucomicrobiae including Akkermansia muciniphila have a LDA score as high as 4.5 in the control group, suggesting significant reduction of this phylum during the progression of fibrosis. Akkermansia muciniphila has been proposed as a biomarker for a healthy gut and shown to link to intestinal health 
(https://pubmed.ncbi.nlm.nih.gov/23671105/). Bacteroidetes and Firmicutes are

287

known key players in gut microbiome for regulating host metabolism and their altered abundance has been proposed to be associated with multiple host immune diseases (https://www.nature.com/articles/s41385-020-0296-4;

https://pubmed.ncbi.nlm.nih.gov/32887946/).

\section{Functional Dynamics in Gut Microbiota in the Mouse Fibrosis Models}

To further elucidate the functional alterations in the gut microbiota of the mouse fibrosis models, we classified the gut microbiome genes into functional categories and compared the abundance of the categories across the four groups of mice. PCoA based on KEGG module abundance revealed a clear separation between Control and Model_7Day/Model_14Day, while the difference between Control and Model_28Day was relatively small, consistent with the clustering pattern inferred from the taxonomic composition (Figure 4A). Analysis of Similarities (ANOSIM) using KEGG orthologous $(\mathrm{KO})$ terms indicated that the abundance of $\mathrm{KO}$ categories are significantly differentiated among the four groups $(p=0.001)$. We presented the top 22 in Figure 4B, including K18197 (rhamnogalacturonan endolyase), K08095 (carboxylic-ester hydrolase), K0771 (AP endonuclease 1), and K16150 (glycogen synthase) among others. A large proportion of the KO categories have elevated abundance in the Model_7Day and/or the Model_14Day.

To unravel the alterations of the biological processes underlying the gut microbiome during the course of mouse fibrosis, we mapped the KO terms to KEGG pathways and compared the pathway abundance among the four groups (Figure 4C 
and 4D). It is shown that the altered pathways are mainly involved in "Metabolism",

“Genetic Information Processing”, and "Cellular Processes”, such as Metabolism of other amino acids, Energy metabolism, Lipid metabolism, Glycan biosynthesis and metabolism, Translation, Transport and catabolism, Cell motility, and Cell community. LEfSe analysis further identified the most representative pathways in each stage of mouse fibrosis, where the Model_7Day is characterized by the pathways of Energy metabolism, Model_14Day contains abundant pathways of Cell community (LDA score $>2.5$ ), and Model_28Day is enriched with pathways related to Translation (Ribosome, LDA score > 3), Glycan biosynthesis and metabolism (LDA score $>3.5$ ), and Lipid metabolism (Terpenoid backbone biosynthesis, LDA score $>2.5$ ) (Figure 4E and Table S3). These remarkable changes in pathways in gut microbiome in mouse fibrosis models highlight the link between the fibrosis progression and functional dynamics of gut microbiota and the multi-faceted functional responses of gut microbiome to host fibrosis.

\section{Correlation between gut microbes and serum metabolites}

To explore the potential relationships between gut microbiota and serum metabolites, we calculated the abundance correlation matrix between the microbiota taxa and serum metabolites (Figure 5). The correlation matrix between gut microbiota taxa and serum metabolites is self-organized to two separate clusters. The first cluster is dominated by taxa from Bacteroidetes (such as Bacteroides_stercoris, Paramuribaculum_intestinale, and Butyricimonas_virosa). Their abundance is positively correlated with the level of diverse GP and two fatty acids Carnitine and 
9,12-octadecadienoic acid (called meta-group-1), while negatively correlated with the

331 level of three other metabolites, i.e., L-Thyroxine, cis-1-Pentadecenoic acid, and

332 N-Methyltryptamine (called meta-group-2). In the contrary, the second cluster mainly

333 comprises of taxa from Firmicutes (such as Clostridioides difficile and Enterococcus

334 faecium) and their abundance correlation with the metabolites exhibits opposite 335 profiles (Figure 5A).

336 Notably, the correlation matrix between gut microbiota $\mathrm{KO}$ categories and serum

337 metabolites is also stratified to two clusters, of which the hierarchical clustering 338 pattern regarding to metabolites is highly in parallel to that for the correlation matrix 339 between gut microbiota taxa and serum metabolites (Figure 5B). Specifically, the first 340 cluster includes two KO terms (K10771- AP endonuclease 1 and K16150-glycogen 341 synthase), which are positively correlated with the meta-group-1. The other $20 \mathrm{KO}$ 342 terms form the second cluster, which are negatively correlated with the meta-group-1 343 and positively correlated with the meta-group-2. Those $20 \mathrm{KO}$ terms are involved in 344 multiple functional modules, such as Transporters, Secretion system, and Metabolism, 345 nonetheless, none of the modules are enriched with the KO terms. The correlation 346 between gut microbiota functions and serum metabolites reveals synergistic dynamic 347 changes between gut microbiota and host metabolism during the progression of 348 fibrosis. The relevant functions associated with the gut microbiota are diverse 349 implying the complexity of the interactions between gut microbiota and host fibrosis. 
with host metabolism in pulmonary fibrosis, we investigated the changes of serum

metabolites and gut microbiome in the mouse fibrosis models during the development of fibrosis. We summarized the main findings in Figure 6. Briefly, the serum metabolic profile and the composition of gut microbes were significantly altered in mice during the progression of fibrosis. The alterations at the two loci exhibit a high level of correlation suggesting a potential link between the serum metabolism and the gut microbiota.

We found that the levels of multiple types of glycerolipids and glycerophospholipids began to decrease significantly after one week of induction and did not return to normal levels until the fourth week. These lipids may help to lubricate the cells of the inner layers of the lung and participate in initiating the repair of damaged tissues leading to the production of pulmonary fibers [26-28]. Abnormal repair after alveolar injury leads to the proliferation of fibrous tissue, which involves complex pathological processes and changes in various lipid metabolites [29-31]. Kuroda et al. found that changes in phospholipid levels in alveolar lavage fluid of patients with pulmonary fibrosis were closely related to the destruction of alveoli and surfactants and the progression of pulmonary fibrosis [32]. Feng Yan et al. showed

370 that the expression levels of glycerophospholipids, which have diagnostic values,

371 were significantly reduced in patients with pulmonary fibrosis by plasma assays [31].

372 We also found that serum concentrations of glycerophospholipids were significantly

373 reduced in the early stages of the bleomycin fibrosis model. The reduced 

generate increased vascular permeability. This not only enhanced lung epithelial cell injury but also further recruited fibroblasts to reach the site of injury. In the late stage

377 of the model, serum glycerophospholipids gradually recovered as vascular 378 permeability decreased.

379 Adenosine is an endogenous purine nucleotide that mostly regulates cyclic 380 adenosine monophosphate (cAMP) levels through adenosine receptors (ARs) and 381 exerts the corresponding physiological effects [33]. In studies of chronic obstructive 382 pulmonary disease and mouse models of lung disease, significantly increased levels of 383 adenosine were observed $[34,35]$. In a bleomycin-induced pulmonary fibrosis model, 384 increased cAMP levels inhibited the overexpression of endothelin in lung tissue and 385 alleviated the symptoms of pulmonary fibrosis, which can directly influence the role of ARs in pulmonary fibrosis [36]. Meanwhile, ARs can induce lung fibroblast differentiation, which is closely related to the release of endothelin-1 and IL-6 from alveolar macrophages and affects the degree of pulmonary fibrosis in mice [37]. In our study, adenosine levels in the model group increased significantly at Model_14Day and started to decrease at Model_28Day, suggesting that adenosine is likely involved in the formation of lung fibrosis in mice and is closely related to the 392 inflammatory response in the early stages of lung fibrosis. Meanwhile, correlation 393 analysis showed that adenosine was positively correlated $(r>0.5)$ with 394 Muribaculaceae_Bacterium_Isolate-114_(HZI), which may be involved in the absorption and transformation of adenosine in the intestinal tract. 

of a variety of diseases (e.g., lymphoma, liver cancer, breast cancer \& pneumonia), but the alteration of gut microbiota in pulmonary fibrosis has rarely been reported [14, 38-43]. Although animal models of fibrosis do not exactly recapitulate IPF, they have yielded many targets for therapeutic intervention [44, 45]. Gen-Cheng Gong et al. performed $16 \mathrm{~S}$ sequencing of gut microbes in a mouse model of pulmonary fibrosis 402 and showed that seven microbes were significantly altered in the pulmonary fibrosis 403 model, including Alloprevotella, Helicobacter, Rikenella, and Rikenllaceae RC9 showed a decreasing trend, and Dubosiella, OIsenella, and Parasutterella showed an increasing trend [17]. Wenjun Li et al. found that in a mouse model of 406 radiation-induced pulmonary fibrosis, the intestinal microbiota was disturbed and 407 intervention to normalize the gut microbiota attenuated pulmonary fibrosis [46]. 408 Ruixue Huang et al. examined the gut microbiota of 18 silicosis patients with severe 409 pulmonary fibrosis and confirmed that Firmicutes and Actinomyces bacteria were 410 significantly reduced in patients with silicosis and progressive pulmonary fibrosis 411 compared to healthy individuals [20]. Here, we detected significantly elevated 412 abundance of Prevotella sp. from Bacteroidetes, Lactobacillus from Firmicutes, and 413 Bifidobacterium from Actinobacteria in the intestine of mice with pulmonary fibrosis.

414 Multiple taxa from Bacteroides, such as Butyricimonas_synergistica and 415 Muribaculaceae, show positive correlation with the cluster dominated by 416 glycerophospholipids, while taxa from Firmicutes, such as Clostridioides difficile and 417 Enterococcus faecium exhibit negative correlation. These results suggest, to some 
extent, that certain gut microbes were altered in parallel with the serum metabolites during the development of pulmonary fibrosis and are likely to be involved in the pathogenesis of the disease, while the specific mechanism of action between pulmonary fibrosis and gut microbes requires subsequent in-depth studies.

By comparing the differential metabolites and microorganisms in Model_7Day, Model_14Day, and Model_28Day with the control group, we found that the number of differential metabolites and microorganisms detected in Model_7Day and Model_14Day was higher than that in Model_28Day. We believe that this phenomenon should be closely related to the pathogenic process of pulmonary fibrosis. At the beginning of the lung fibrosis model, due to the presence of harmful substances, lung fibroblasts were activated and secreted a large amount of extracellular matrix, and the subsequent production of cytokines mediated the proliferation and differentiation of fibroblasts by macrophages approaching the damaged site $[1,8]$. As a result, a large number of differential metabolites associated with inflammation appear in the serum during this period. In addition, the gut microbiota is closely related to the immune status of the host $[47,48]$. In the later stages of pulmonary fibrosis, the lungs are in a state of the tissue and vascular repair and remodeling, the inflammatory response decreases, the inflammation-related metabolites present in the serum gradually decrease, the fibrosis-related metabolites in the lung tissue begin to increase, and the gut microbiota gradually returns to normal, but still maintains some of the characteristics of the pulmonary fibrosis period. 
The advantage of this study is that we applied metagenome sequencing and metabolomics technology, which allowed us to obtain the information about the host-gut microbiota-metabolism interaction in response to the development of pulmonary fibrosis. The limitation of this study is the small sample size, and therefore, further large cohorts are needed to confirm the alterations and to determine the causal relationship between gut microbiota and serum metabolites in human samples. Despite these limitations, the present study opens new avenues for understanding the association between gut microbiota and serum metabolitesin pulmonary fibrosis models.

\section{Consent for publication}

Not applicable.

\section{Funding}

This work was funded by grants from the Natural Science Founding of Shandong Province (ZR202103030823) and the National Natural Science Foundation of China (81901321, 82000268).

\section{Conflicts of interest}

No conflicts of interest, financial or otherwise, are declared by the authors. Author Hui Zheng was employed by the company Wuhan Metware Biotechnology Co., Ltd.. The remaining authors declare that the research was conducted in the absence of any commercial or financial relationships that could be construed as a potential conflict of interest. 


\section{Availability of data and materials}

462 All data generated or analyzed during this study are included in this published article 463 and supplementary files..

464

465

\section{Ethics approval and consent to participate}

All experimental procedures and methods were carried out in accordance with the Guide for the Care and Use of Laboratory Animals provided by the institutional Animal Care and Use Advisory Committee for Qingdao University. The study was carried out in compliance with the ARRIVE guidelines. All animal experimental protocols used in this study were approved by the Ethics Committee of Affiliated Hospital of Qingdao University (Ethics NO. QYFYW2LL26275).

\section{Authors' contributions}

X.H.Y., X.J.J., and X.J.J. conceived and designed research; X.H.Y, X.S.C., X.M.W., and Y.Z.Z. performed experiments; X.H.Y., F.F.W., J.P.C., H.Y.W., and Y.Z.Z. analyzed data; X.H.Y., H.Y.W, H.Z., and Y.Z.Z. interpreted results of experiments; X.H.Y., X.S.C., F.F.W., H.Y.W., and Y.Z.Z. prepared figures; X.H.Y., X.J.J., X.M.W., J.P.C., H.Z., and Y.Z.Z. drafted manuscript; X.H.Y., X.J.J., H.Z., and Y.Z.Z. edited and revised manuscript; X.H.Y., X.J.J., and Y.Z.Z. approved final version of the manuscript.

\section{Acknowledgements}

We are grateful to Metware Biotechnology Co., Ltd for support on UPLC-MS experiments and data acquisition. 


\section{References}

1. Noble PW, Barkauskas CE, Jiang D (2012) Pulmonary fibrosis: patterns and perpetrators. The Journal of clinical investigation 122 (8):2756-2762. doi: 10.1172/JCl60323.

2. Wynn TA, Ramalingam TR (2012) Mechanisms of fibrosis: therapeutic translation for fibrotic disease. Nat Med 18 (7):1028-1040. doi: 10.1038/nm.2807.

3. King TE, Jr., Albera C, Bradford WZ, Costabel U, Hormel P, Lancaster L, Noble PW, Sahn SA, Szwarcberg J, Thomeer M, Valeyre D, du Bois RM, Group IS (2009) Effect of interferon gamma-1b on survival in patients with idiopathic pulmonary fibrosis (INSPIRE): a multicentre, randomised, placebo-controlled trial. Lancet 374 (9685):222-228. doi: 10.1016/S0140-6736(09)60551-1.

4. Fernandez IE, Eickelberg $\mathrm{O}$ (2012) New cellular and molecular mechanisms of lung injury and fibrosis in idiopathic pulmonary fibrosis. Lancet 380 (9842):680 -688. doi: 10.1016/S0140-6736(12)61144-1.

5. Martinez FJ, Collard HR, Pardo A, Raghu G, Richeldi L, Selman M, Swigris JJ, Taniguchi H, Wells AU (2017) Idiopathic pulmonary fibrosis. Nature reviews Disease primers 3:17074. doi: 10.1038/nrdp.2017.74.

6. Kropski JA, Blackwell TS (2019) Progress in Understanding and Treating Idiopathic Pulmonary Fibrosis. Annual review of medicine 70:211-224. doi: 10.1146/annurev-med-041317-102715.

7. Heukels P, Moor CC, von der Thüsen JH, Wijsenbeek MS, Kool M (2019) Inflammation and immunity in IPF pathogenesis and treatment. Respiratory medicine 147:79-91. doi: 10.1016/j.rmed.2018.12.015.

8. Thannickal VJ, Toews GB, White ES, Lynch JP, 3rd, Martinez FJ (2004) Mechanisms of pulmonary fibrosis. Annual review of medicine 55:395-417. doi: 10.1146/annurev.med.55.091902.103810.

9. Hewlett JC, Kropski JA, Blackwell TS (2018) Idiopathic pulmonary fibrosis: Epithelial-mesenchymal interactions and emerging therapeutic targets. Matrix biology: journal of the International Society for Matrix Biology 71-72:112-127. doi: 10.1016/j.matbio.2018.03.021.

10. Xie N, Tan Z, Banerjee S, Cui H, Ge J, Liu RM, Bernard K, Thannickal VJ, Liu G (2015) Glycolytic Reprogramming in Myofibroblast Differentiation and Lung Fibrosis. Am J Respir Crit Care Med 192 (12):1462-1474. doi: 10.1164/rccm.201504-07800C.

11. Xie N, Cui H, Ge J, Banerjee S, Guo S, Dubey S, Abraham E, Liu RM, Liu G (2017) Metabolic characterization and RNA profiling reveal glycolytic dependence of profibrotic phenotype of alveolar macrophages in lung fibrosis. Am J Physiol Lung Cell Mol Physiol 313 (5):L834 -L844. doi: 10.1152/ajplung.00235.2017.

12. Kang YP, Lee SB, Lee JM, Kim HM, Hong JY, Lee WJ, Choi CW, Shin HK, Kim DJ, Koh ES, Park CS, Kwon SW, Park SW (2016) Metabolic Profiling Regarding Pathogenesis of Idiopathic Pulmonary Fibrosis. Journal of proteome research 15 (5):1717-1724. doi: 10.1021/acs.jproteome.6b00156. 
13. Rindlisbacher B, Schmid C, Geiser T, Bovet C, Funke-Chambour M (2018) Serum metabolic profiling identified a distinct metabolic signature in patients with idiopathic pulmonary fibrosis - a potential biomarker role for LysoPC. Respiratory research 19 (1):7. doi: 10.1186/s12931-018-0714-2.

14. Schuijt TJ, Lankelma JM, Scicluna BP, de Sousa e Melo F, Roelofs JJ, de Boer JD, Hoogendijk AJ, de Beer R, de Vos A, Belzer C, de Vos WM, van der Poll T, Wiersinga WJ (2016) The gut microbiota plays a protective role in the host defence against pneumococcal pneumonia. Gut 65 (4):575-583. doi: 10.1136/gutjnl-2015-309728.

15. Liu C, Yang L, Han Y, Ouyang W, Yin W, Xu F (2019) Mast cells participate in regulation of lung-gut axis during Staphylococcus aureus pneumonia. Cell Prolif 52 (2):e12565. doi: 10.1111/cpr.12565.

16. Arrieta MC, Stiemsma LT, Dimitriu PA, Thorson L, Russell S, Yurist-Doutsch S, Kuzeljevic B, Gold MJ, Britton HM, Lefebvre DL, Subbarao P, Mandhane P, Becker A, McNagny KM, Sears MR, Kollmann T, Investigators CS, Mohn WW, Turvey SE, Finlay BB (2015) Early infancy microbial and metabolic alterations affect risk of childhood asthma. Sci Transl Med 7 (307):307ra152. doi: 10.1126/scitransImed.aab2271.

17. Gong GC, Song SR, Su J (2021) Pulmonary fibrosis alters gut microbiota and associated metabolites in mice: An integrated 16S and metabolomics analysis. Life sciences 264:118616. doi: 10.1016/j.lfs.2020.118616.

18. Biedermann L, Brulisauer K, Zeitz J, Frei P, Scharl M, Vavricka SR, Fried M, Loessner MJ, Rogler G, Schuppler M (2014) Smoking cessation alters intestinal microbiota: insights from quantitative investigations on human fecal samples using FISH. Inflamm Bowel Dis 20 (9):1496-1501. doi: 10.1097/MIB.0000000000000129.

19. Felix KM, Jaimez IA, Nguyen TV, Ma H, Raslan WA, Klinger CN, Doyle KP, Wu HJ (2018) Gut Microbiota Contributes to Resistance Against Pneumococcal Pneumonia in Immunodeficient Rag(-/-) Mice. Front Cell Infect Microbiol 8:118. doi: 10.3389/fcimb.2018.00118.

20. Zhou Y, Chen L, Sun G, Li Y, Huang R (2019) Alterations in the gut microbiota of patients with silica-induced pulmonary fibrosis. Journal of occupational medicine and toxicology (London, England) 14:5. doi: 10.1186/s12995-019-0225-1.

21. Kapur R, Kim M, Rebetz J, Hallstrom B, Bjorkman JT, Takabe-French A, Kim N, Liu J, Shanmugabhavananthan S, Milosevic S, McVey MJ, Speck ER, Semple JW (2018) Gastrointestinal microbiota contributes to the development of murine transfusion-related acute lung injury. Blood Adv 2 (13):1651-1663. doi: 10.1182/bloodadvances.2018018903.

22. Quehenberger O, Armando AM, Brown AH, Milne SB, Myers DS, Merrill AH, Bandyopadhyay S, Jones KN, Kelly S, Shaner RL, Sullards CM, Wang E, Murphy RC, Barkley RM, Leiker TJ, Raetz CR, Guan Z, Laird GM, Six DA, Russell DW, McDonald JG, Subramaniam S, Fahy E, Dennis EA (2010) Lipidomics reveals a remarkable diversity of lipids in human plasma. Journal of lipid research 51 (11):3299-3305. doi: 10.1194/jlr.M009449.

23. Xuan Q, Hu C, Yu D, Wang L, Zhou Y, Zhao X, Li Q, Hou X, Xu G (2018) Development of a High Coverage Pseudotargeted Lipidomics Method Based on Ultra-High Performance Liquid Chromatography-Mass Spectrometry. Analytical chemistry 90 (12):7608-7616. doi: 10.1021/acs.analchem.8b01331.

24. Tang S, Borodovsky M (2015) Ab Initio Gene Identification in Metagenomic Sequences. In: Nelson KE (ed) Encyclopedia of Metagenomics: Genes, Genomes and Metagenomes: Basics, 
Methods, Databases and Tools. Springer US, Boston, MA, pp 13-19.doi: 10.1007/978-1-4614-6418-1_440-1.

25. Paulson JN, Stine OC, Bravo HC, Pop M (2013) Differential abundance analysis for microbial marker-gene surveys. Nature Methods 10 (12):1200-1202. doi: 10.1038/nmeth.2658.

26. Romero F, Shah D, Duong M, Penn RB, Fessler MB, Madenspacher J, Stafstrom W, Kavuru M, Lu B, Kallen CB, Walsh K, Summer R (2015) A pneumocyte-macrophage paracrine lipid axis drives the lung toward fibrosis. American journal of respiratory cell and molecular biology 53 (1):74-86. doi: 10.1165/rcmb.2014-03430C.

27. Gwyer Findlay E, Hussell T (2012) Macrophage-mediated inflammation and disease: a focus on the lung. Mediators of inflammation 2012:140937. doi: 10.1155/2012/140937.

28. Suryadevara V, Ramchandran R, Kamp DW, Natarajan V (2020) Lipid Mediators Regulate Pulmonary Fibrosis: Potential Mechanisms and Signaling Pathways. International journal of molecular sciences 21 (12). doi: 10.3390/ijms21124257.

29. Du XG, Ruan XZ (2019) Lipid Metabolism Disorder and Renal Fibrosis. Advances in experimental medicine and biology 1165:525-541. doi: 10.1007/978-981-13-8871-2_26.

30. Summer R, Mora AL (2019) Lipid Metabolism: A New Player in the Conundrum of Lung Fibrosis. American journal of respiratory cell and molecular biology 61 (6):669-670. doi: 10.1165/rcmb.2019-0098ED.

31. Yan F, Wen Z, Wang R, Luo W, Du Y, Wang W, Chen X (2017) Identification of the lipid biomarkers from plasma in idiopathic pulmonary fibrosis by Lipidomics. BMC pulmonary medicine 17 (1):174. doi: 10.1186/s12890-017-0513-4.

32. Kuroda K, Morimoto Y, Ogami A, Oyabu T, Nagatomo H, Hirohashi M, Yamato H, Nagafuchi Y, Tanaka I (2006) Phospholipid concentration in lung lavage fluid as biomarker for pulmonary fibrosis. Inhalation toxicology 18 (5):389-393. doi: 10.1080/08958370500516200.

33. Phan THG, Paliogiannis $P$, Nasrallah GK, Giordo R, Eid AH, Fois AG, Zinellu A, Mangoni AA, Pintus $G$ (2021) Emerging cellular and molecular determinants of idiopathic pulmonary fibrosis. Cellular and molecular life sciences : CMLS 78 (5):2031-2057. doi: 10.1007/s00018-020-03693-7.

34. Schneider DJ, Lindsay JC, Zhou Y, Molina JG, Blackburn MR (2010) Adenosine and osteopontin contribute to the development of chronic obstructive pulmonary disease. FASEB journal : official publication of the Federation of American Societies for Experimental Biology 24 (1):70-80. doi: 10.1096/fj.09-140772.

35. Blackburn MR, Lee CG, Young HW, Zhu Z, Chunn JL, Kang MJ, Banerjee SK, Elias JA (2003) Adenosine mediates IL-13-induced inflammation and remodeling in the lung and interacts in an IL-13-adenosine amplification pathway. The Journal of clinical investigation 112 (3):332-344. doi: 10.1172/JCl16815.

36. Zhou Y, Schneider DJ, Morschl E, Song L, Pedroza M, Karmouty-Quintana H, Le T, Sun CX, Blackburn MR (2011) Distinct roles for the A2B adenosine receptor in acute and chronic stages of bleomycin-induced lung injury. Journal of immunology (Baltimore, Md : 1950) 186 (2):1097-1106. doi: 10.4049/jimmunol.1002907.

37. Zhou Y, Murthy JN, Zeng D, Belardinelli L, Blackburn MR (2010) Alterations in adenosine metabolism and signaling in patients with chronic obstructive pulmonary disease and idiopathic pulmonary fibrosis. PloS one 5 (2):e9224. doi: 10.1371/journal.pone.0009224.

38. Yamamoto ML, Schiestl RH (2014) Lymphoma caused by intestinal microbiota. International 
journal of environmental research and public health 11 (9):9038-9049. doi: 10.3390/ijerph110909038.

39. Yu LX, Schwabe RF (2017) The gut microbiome and liver cancer: mechanisms and clinical translation. Nature reviews Gastroenterology \& hepatology 14 (9):527-539. doi: 10.1038/nrgastro.2017.72.

40. Mima K, Nakagawa S, Sawayama H, Ishimoto T, Imai K, Iwatsuki M, Hashimoto D, Baba Y, Yamashita YI, Yoshida N, Chikamoto A, Baba H (2017) The microbiome and hepatobiliary-pancreatic cancers. Cancer letters 402:9-15. doi: 10.1016/j.canlet.2017.05.001.

41. Chen J, Douglass J, Prasath V, Neace M, Atrchian S, Manjili MH, Shokouhi S, Habibi M (2019) The microbiome and breast cancer: a review. Breast cancer research and treatment 178 (3):493-496. doi: 10.1007/s10549-019-05407-5.

42. Kwa M, Plottel CS, Blaser MJ, Adams S (2016) The Intestinal Microbiome and Estrogen Receptor-Positive Female Breast Cancer. Journal of the National Cancer Institute 108 (8) .doi: 10.1093/jnci/djw029.

43. Wypych TP, Wickramasinghe LC, Marsland BJ (2019) The influence of the microbiome on respiratory health. Nature immunology 20 (10):1279-1290. doi: 10.1038/s41590-019-0451-9.

44. Peyser R, MacDonnell S, Gao Y, Cheng L, Kim Y, Kaplan T, Ruan Q, Wei Y, Ni M, Adler C, Zhang W, Devalaraja-Narashimha K, Grindley J, Halasz G, Morton L (2019) Defining the Activated Fibroblast Population in Lung Fibrosis Using Single-Cell Sequencing. American journal of respiratory cell and molecular biology 61 (1):74-85. doi: 10.1165/rcmb.2018-03130C.

45. Della Latta V, Cecchettini A, Del Ry S, Morales MA (2015) Bleomycin in the setting of lung fibrosis induction: From biological mechanisms to counteractions. Pharmacological research 97:122-130. doi: 10.1016/j.phrs.2015.04.012.

46. Li W, Lu L, Liu B, Qin S (2020) Effects of phycocyanin on pulmonary and gut microbiota in a radiation-induced pulmonary fibrosis model. Biomedicine \& pharmacotherapy $=$ Biomedecine \& pharmacotherapie 132:110826. doi: 10.1016/j.biopha.2020.110826.

47. Rooks MG, Garrett WS (2016) Gut microbiota, metabolites and host immunity. Nature reviews Immunology 16 (6):341-352. doi: 10.1038/nri.2016.42.

48. Pickard JM, Zeng MY, Caruso R, Núñez G (2017) Gut microbiota: Role in pathogen colonization, immune responses, and inflammatory disease. Immunological reviews 279 (1):70-89. doi: 10.1111/imr.12567.

\section{FIGURE LEGENDS}

\section{Figure 1 Bleomycin mouse models.}

(A) Flow chart of Bleomycin mouse models, collecting feces and serum from 7 th 
653

654

655

656

657

658

659

660

661

662

663

664

665

666

667

668

669

670

671

672

673

674

day,14th day to 28th day after pulmonary fibrosis induced; (B) TGF- $\beta$ and a-SMA expression of mouse lung tissues at 7, 14, and 28 days after bleomycin instillation; (C) Lung histopathology after a single dose of bleomycin treatment (HE and Masson staining). Representative hematoxylin- and eosin-stained (a, b, c, and d) and Masson's trichrome-stained (e, f, g, and h) lung tissue sections are shown. The mice were sacrificed at 7 (b and f), 14 (c and g), and 28 (d and h) days after bleomycin instillation. Except for the PBS control (a and e), all panels showed lung sections from bleomycin-treated mice. The lungs were inflated with $10 \%$ buffered formaldehyde. Original magnification, 5.0X; insets: 20X; (D) The results of TGF- $\beta$ immunohistochemistry. Positive Area Ratio, reflecting the proportion of the positive area.

\section{Figure 2 Metabolome profiling of serum.}

(A) The multivariate statistical model of the Control, Model_7Day, Model_14Day, and Model_28Day groups. Principal component analysis (PCA) of Control, Model_7Day, Model_14Day, and Model_28Day (R2X=0.617, Q2=0.409); The clustering analyses of orthogonal partial least-squares discriminant analysis (OPLS-DA) of Control and Model_7Day group (R2X=0.614, R2Y=1, Q2=0.944), Control and Model_14Day group (R2X=0.59, R2Y=1, Q2=0.928), and Control and Model_28Day group (R2X=0.349, R2Y $=0.99, \mathrm{Q} 2=0.527)$. (B) Relative abundance of representative differential serum metabolites in three groups, as compared to that of respective Control group. GP, glycerophospholipids; GL, glycerolipids; FA, fatty 
acyls;(C) KEGG pathway analysis, the color of the point represents the $p$-value, the size of the point represents the enrichment factor, and the shape of the point reprents the changes in metabolic pathways; (D) The changes of four metabolic pathways in pulmonary fibrosis mice at different time periods.

Figure 3 Species difference analysis of fecal microbes.

(A) Alpha diversity analysis (Shannon diversity index) among the four groups at the species level; (B) Principal coordinate analysis (PCoA) of the fecal microbiota; (C) LDA scores for the bacterial taxa differentially abundant among Control, Model_7Day, Model_14Day, and Model_28Day.

\section{Figure 4 Fecal microbes functional composition and difference analysis on} KEGG.

(A) Functional PCoA based on the Bray-Curtis distances of fecal microbes; (B) Heatmap of TOP22 differential functional units, red represents higher abundance in corresponding samples $\mathrm{KO}$, blue represents lower abundance $\mathrm{KO}$; (C) The histogram of the groups KEGG primary metabolic pathways; (D) The histogram of each group KEGG secondary metabolic pathway; (E) LEfSe functional branch diagram, showing the topological relationship of the main functional groups in the sample community from KEGG pathway level 1 to level 3( from inner to outer).

\section{Figure 5 Correlation analysis of metabolites and fecal microbes.}

(A) Spearman's rank correlation between differential gut microbial species and 
metabolites, 40 samples were used for Spearman's rank correlation, (B) Spearman's

698 rank correlation between differential gut microbial functional $\mathrm{KO}$ and metabolites.

699 Red, represent positive correlations; blue, represent negative correlations.

700

Figure 6 Graphical summary of the key results of the study.

702

703

704

705

706

707

708

709

710

711

712

713

714

715

716

717

The time-dependent changes of major serum metabolites are indicated as trend lines.

The representative gut microbiome and the pathways at each time points are shown explicitly.

Figures are reproduced or adapted from www.wikidata.org Creative Common BY 3.0) , www.creationwiki.org (Creative Common Attribution-ShareAlike License), or www. wikidata.org (Creative Commons CC0 License).

\section{TABLE LEGENDS}

Table S1 Screening for differential metabolites among Model_7Day, Model_14Day, Model_28Day, and Control.

Table S2 Representative differential metabolites among Model_7Day, Model_14Day, Model_28Day, and Control.

Table S3 LEfSe (LDA effect size) analysis of all samples identified 79 taxa.

Table S4 Representative differential gut microbes functional unit in all samples. 
Figures

A

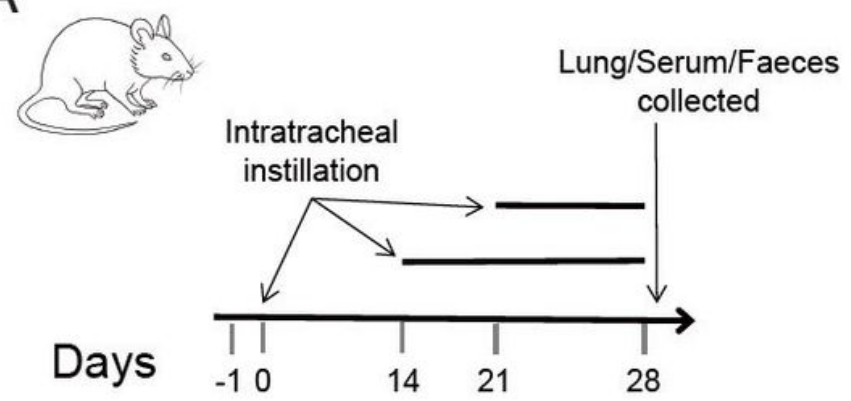

C

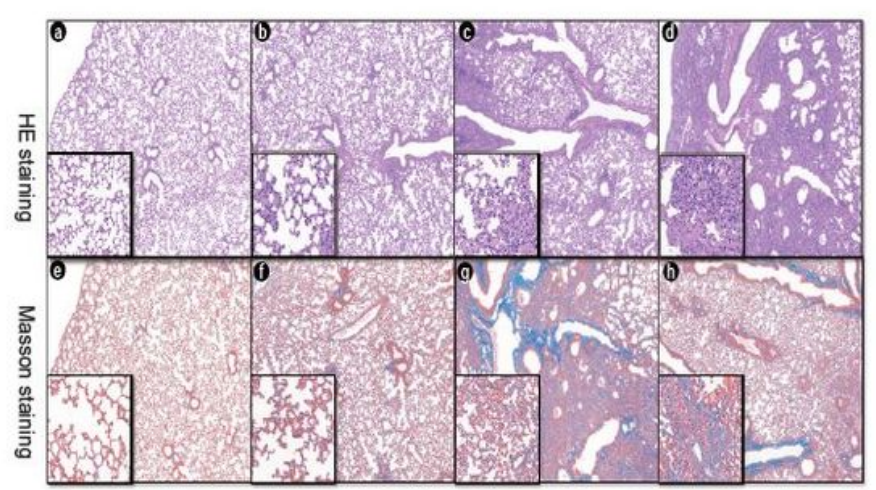

B

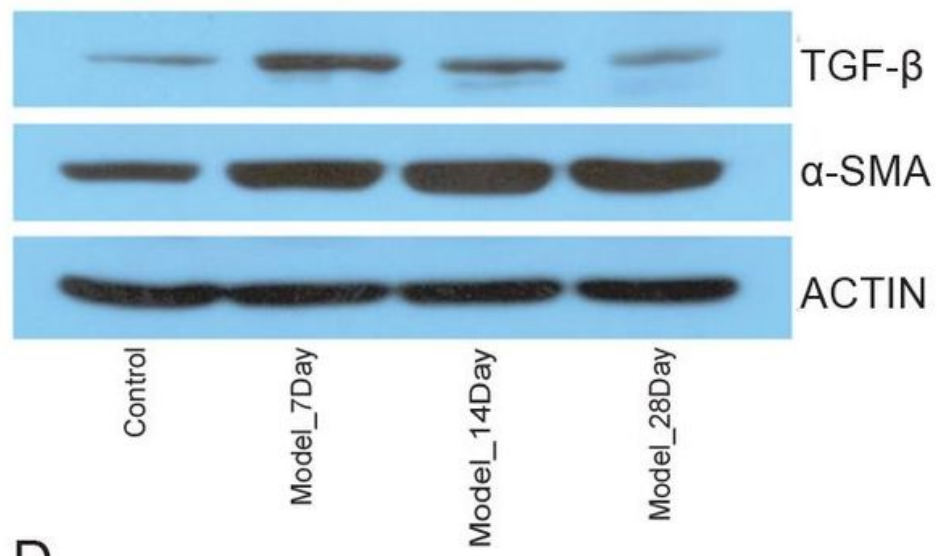

D

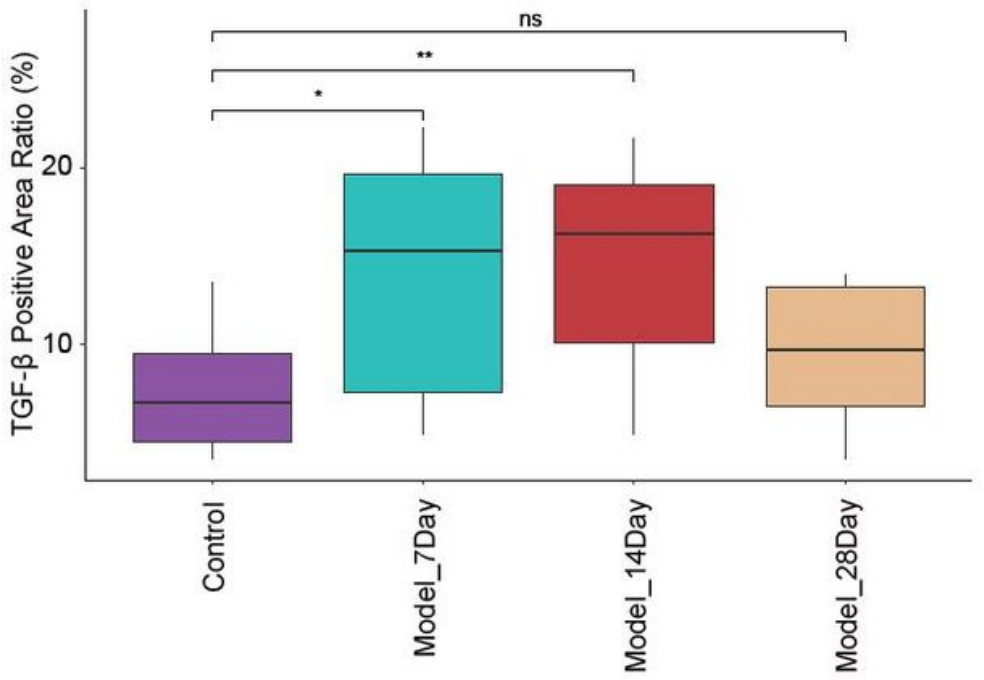

Figure 1

a single dose of bleomycin treatment (HE and Masson staining). Representative hematoxylin and eosin stained ( $a, b, c$, and $d)$ and Masson's trichrome stained $(e, f, g$, and $h$ ) lung tissue sections are shown. The mice were sacrificed at 7 ( $b$ and $f), 14$ ( $c$ and $g$ ), and 28 ( $d$ and $h$ ) days after bleomycin instillation. Except for the PBS control (a and e), all panels showed lung sections from bleomycin treated mice. The lungs were inflated with $10 \%$ buffered formaldehyde. Original magnification, 5.0X; insets: 20X; (D) The results of TGF $\beta$ immunohistochemistry. Positive Area Ratio, reflecting the proportion of the positive area. 
A
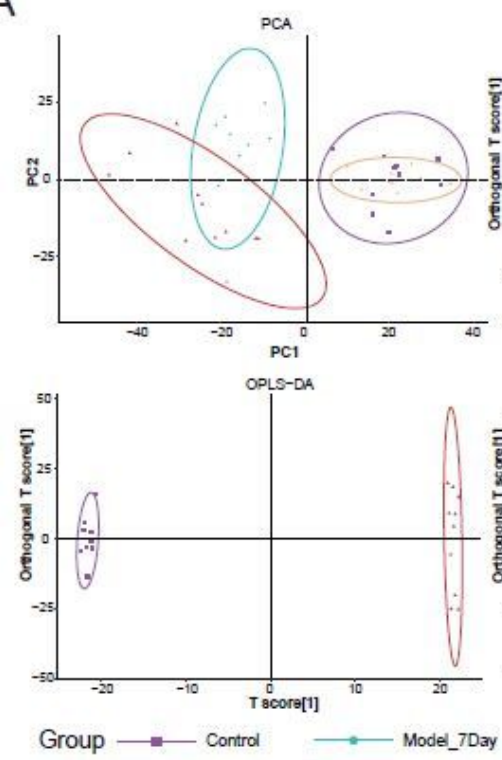

C

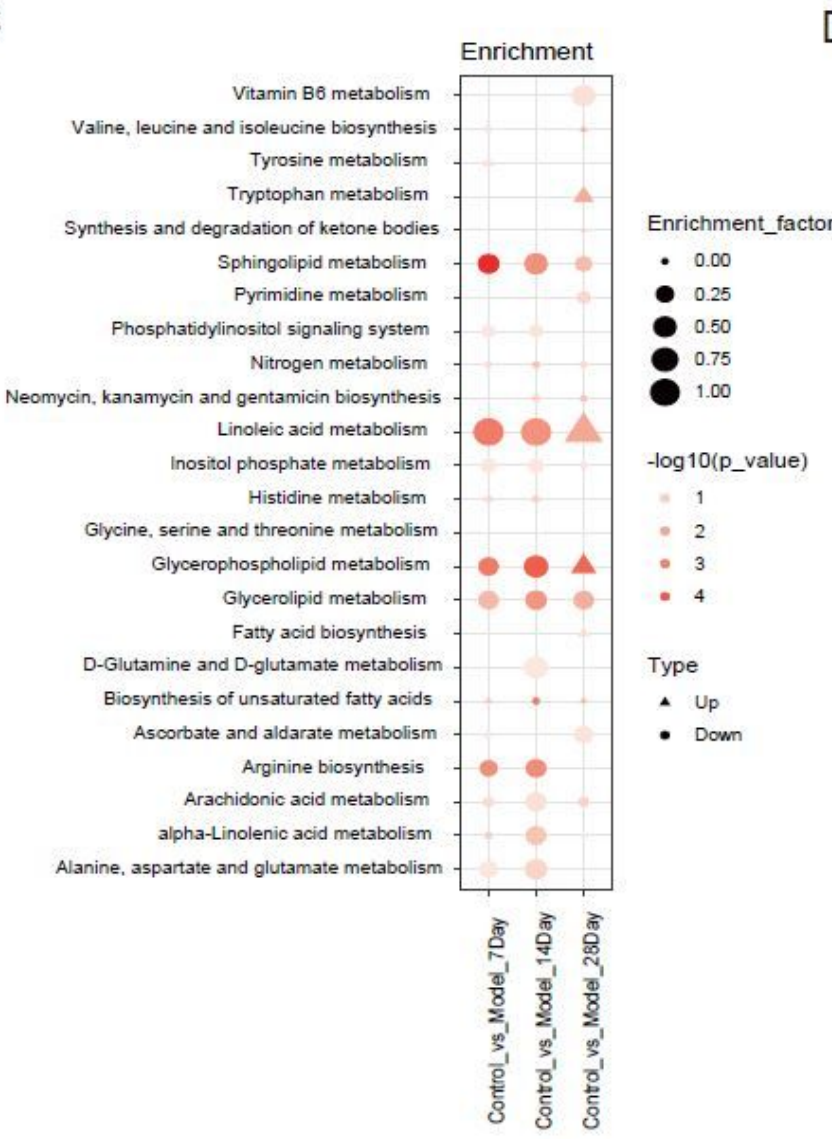

B
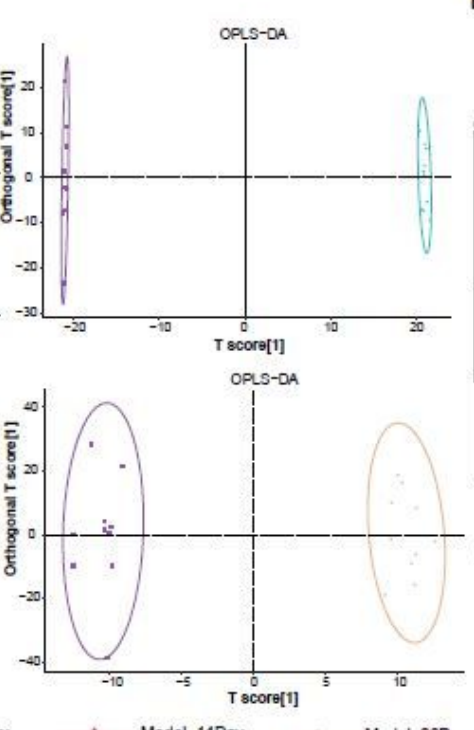

D
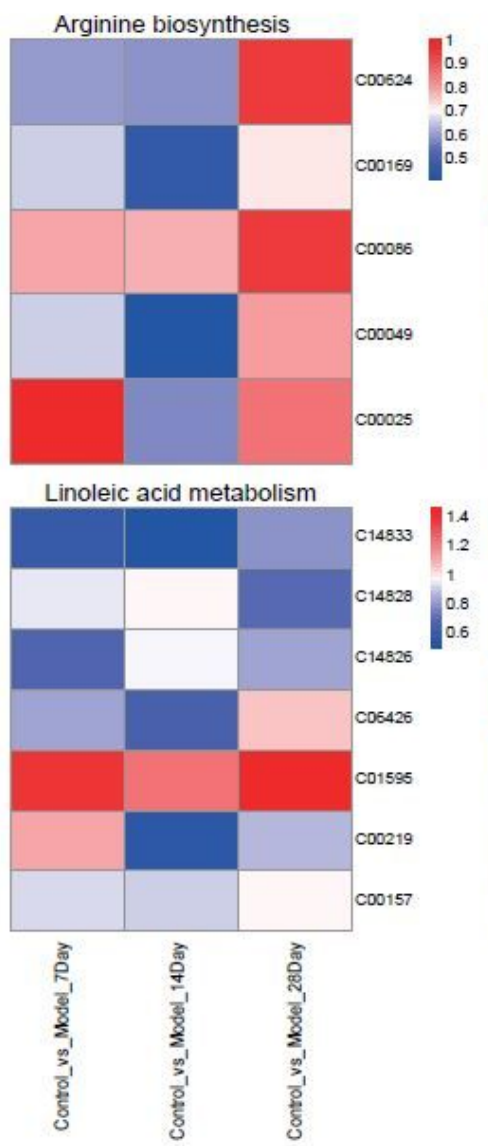

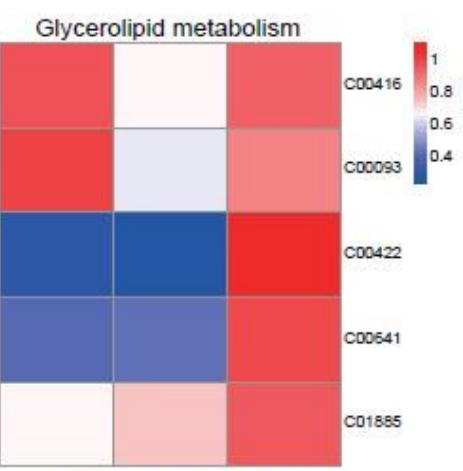

Sphingolipid metabolism

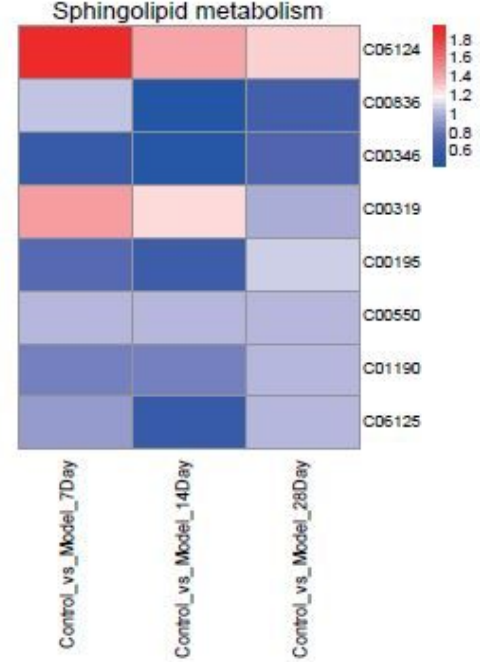

\section{Figure 2}

Metabolome profiling of serum. (A) The multivariate statistical model of the Control, Model_7Day, Model_14Day, and Model_28Day groups. Principal component analysis (PCA) of Control, Model_7Day, Model_14Day, and Model_28Day (R2X=0.617, Q2=0.409); The clustering analyses of orthogonal partial least squares discriminant analysis (OPLS DA) of Control and Model_7Day group ( $R 2 X=0.614, R 2 Y=1$, $\mathrm{Q} 2=0.944)$, Control and Model_14Day group (R2X=0.59, R2Y=1, Q2=0.928), and Control and Model_28Day group ( $R 2 X=0.349, R 2 Y=0.99, Q 2=0.527)$. (B) Relative abundance of representative 
differential serum metabolites in three groups, as compared to that of respective Control group. GP, glycerophospholipids; GL, glycerolipids; FA, fatty acyls;(C) KEGG pathway analysis, the color of the point represents the $p$-value, the size of the point represents the enrichment factor, and the shape of the point reprents the changes in metabolic pathways; (D) The changes of four metabolic pathways in pulmonary fibrosis mice at different time periods.
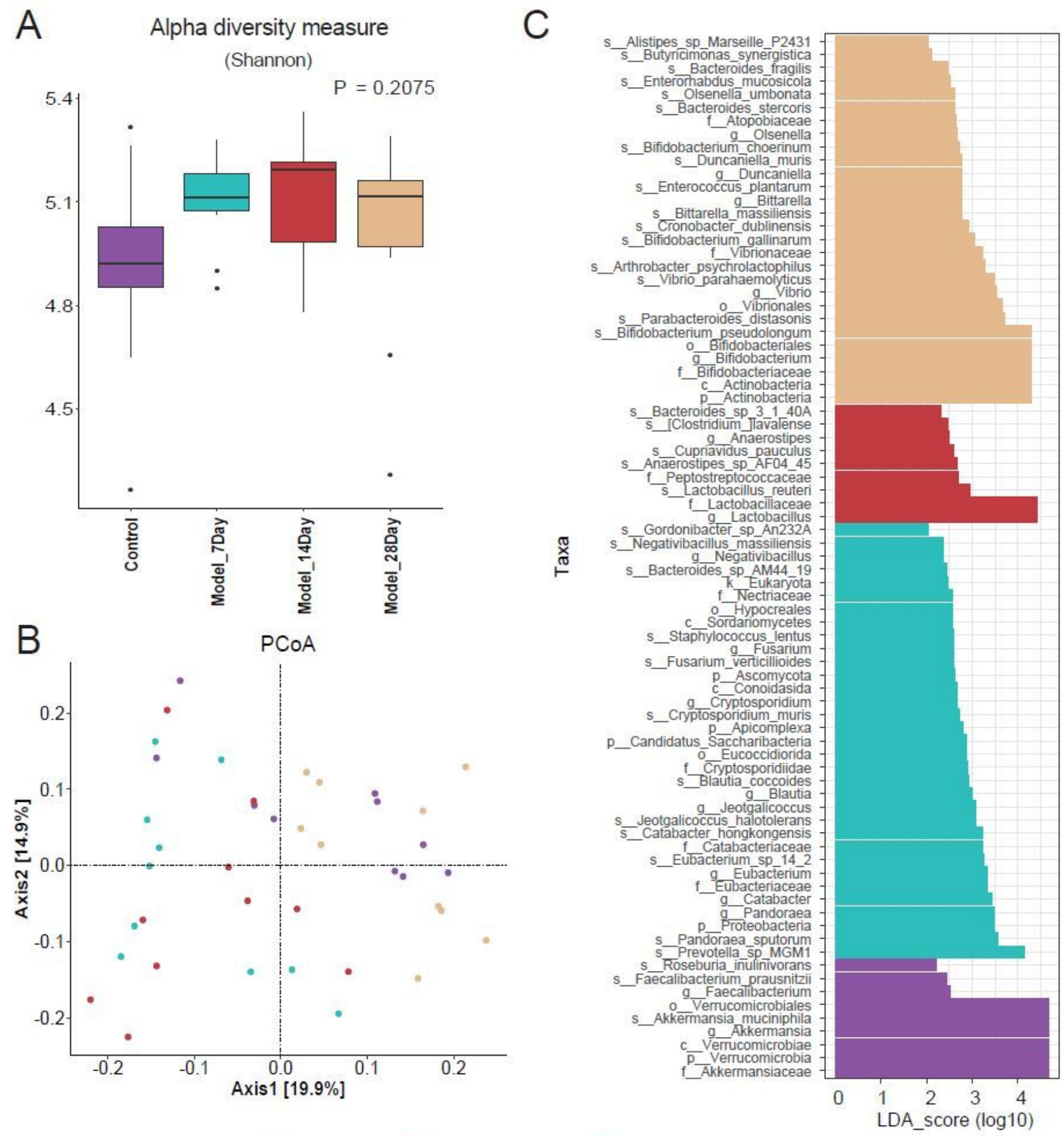

Group

Control

Model_7Day

Model_14Day

Model_28Day 
Species difference analysis of fecal microbes. (A) Alpha diversity analysis (Shannon diversity index) among the four groups at the species level; (B) Principal coordinate analysis (PCoA) of the fecal microbiota; (C) LDA scores for the bacterial taxa differentially abundant among Control, Model_7Day, Model_14Day, and Model_28Day.

A

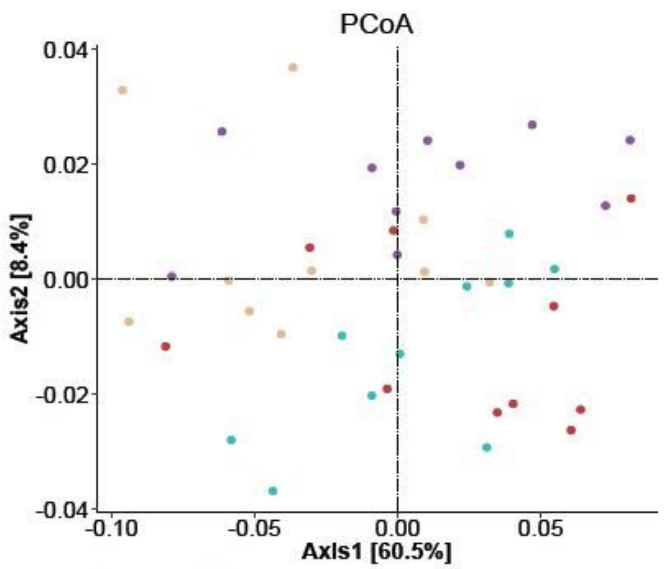

Group Control Model_7Day Model_14Day Model_28Day

C

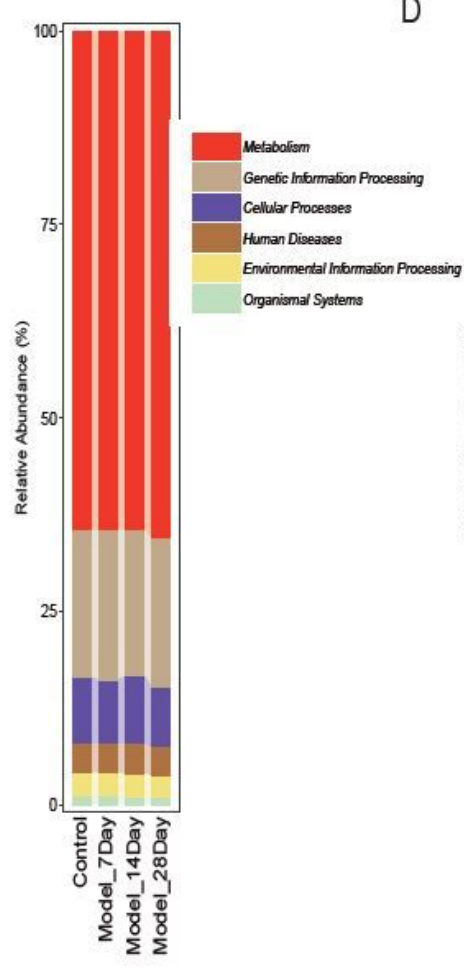

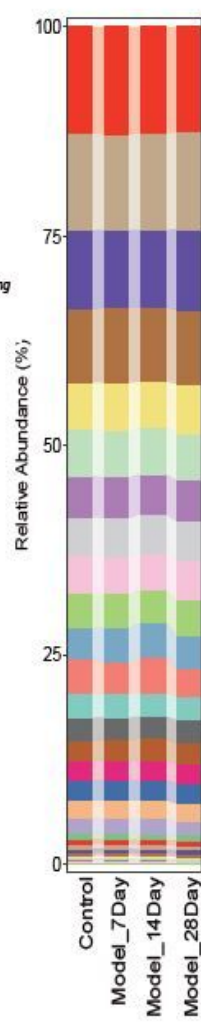

B

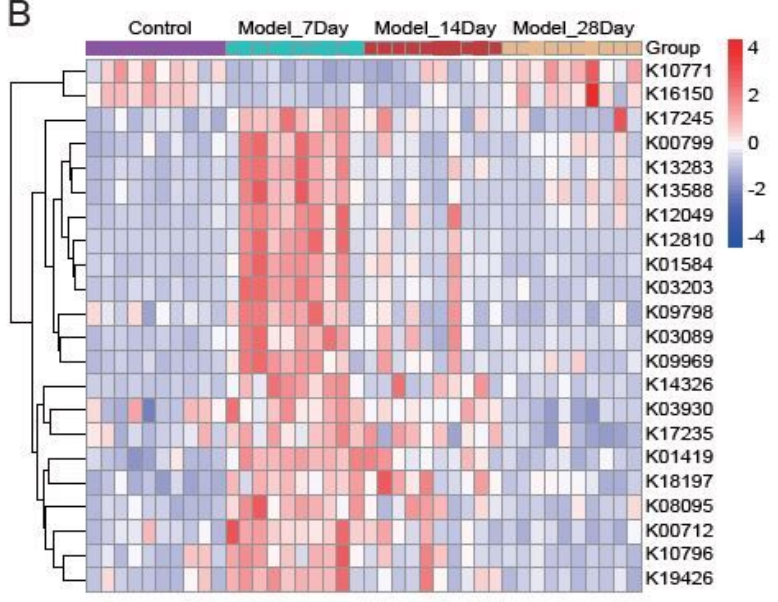

E

Metaboilsm; Carbohycrate metabolis Metabolism; Amino acid metabolism Genetic Information Processing.Replication and repair Genetic Information Processing, Transalation Metabolism; Metabalism of other amino acids Metabolism, Energy metabolism Metabolism; Lipid metabolism Genetic information Processing, Folding, sorting and degradation Metabolism; Biosynthesis of other secondary metabolites Cellibar Processes, Cell motility Human Diseases; Drug resistance: antimicrobial Metabolism; Metabolism of terpenaids and polykefides Metabolism; Xenobiodics biodegradation and metabolism

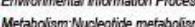
Cellitar Processes; Cell growth and deat Cellitiar Processes, Celilular community - prokanyotes Human Diseases, infectious disease: bacterial Human Diseases; hrfectious disease: bacterial
Genetic information Processing, Transcripfion Envelic information Processing, Tansecription Eenvionnmental Information Processing, Signal Processes; Transport and catabolism Organismal Systems,Emvirommental adaptation Organismal Systems:Aging Organismal Systems:Endocine system Organismal Systems;:Digestive system Human Diseases, Neurodegenerative disease Organismal Systemsimmune system
Human Diseases; Cancer, overview Human Diseases, infectious disease: parasibc Human Diseases, Drug resistance: antineoplastic Organiamal Syotems;:Development and regeneration Human Diseases, Infectious disease: viral Human Disesses; Gactious disease: via

\section{Figure 4}

Fecal microbes functional composition and difference analysis on 685 KEGG. (A) Functional PCoA based on the Bray Curtis distances of fecal microbes; (B) Heatmap of TOP22 differential functional units, red represents higher abundance in corresponding samples KO, blue represents lower abundance KO; (C) The histogram of the groups KEGG primary metabolic pathways; (D) The histogram of each group KEGG secondary metabolic pathway; (E) LEfSe functional branch diagram, showing the topological relationship 
of the main functional groups in the sample community from KEGG pathway level 1 to level 3 (from inner to outer).

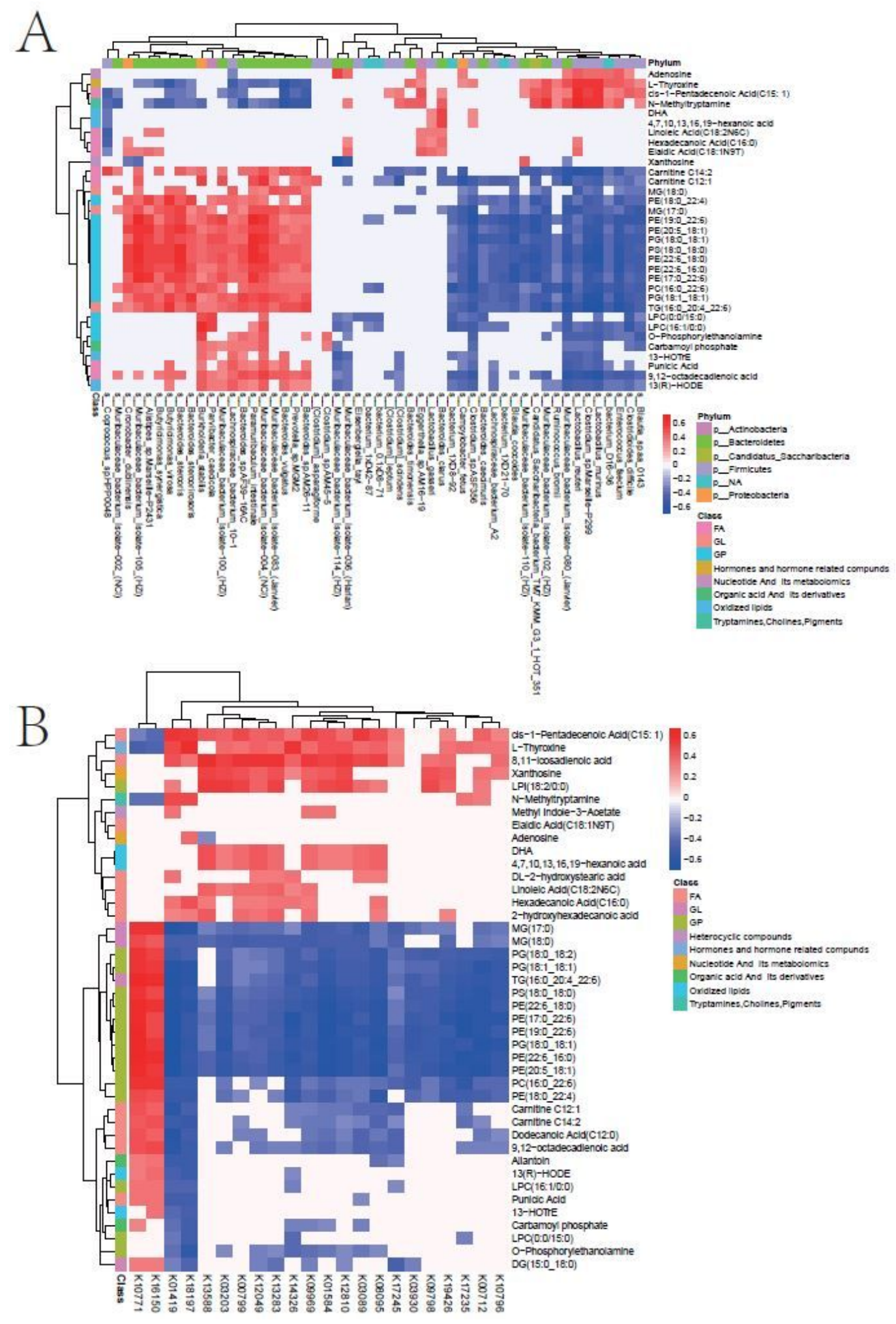

Figure 5

Correlation analysis of metabolites and fecal microbes. (A) Spearman's rank correlation between differential gut microbial species and metabolites, 40 samples were used for Spearman's rank correlation, 
(B) Spearman's rank correlation between differential gut microbial functional KO and metabolites. Red, represent positive correlations; blue, represent negative correlations.

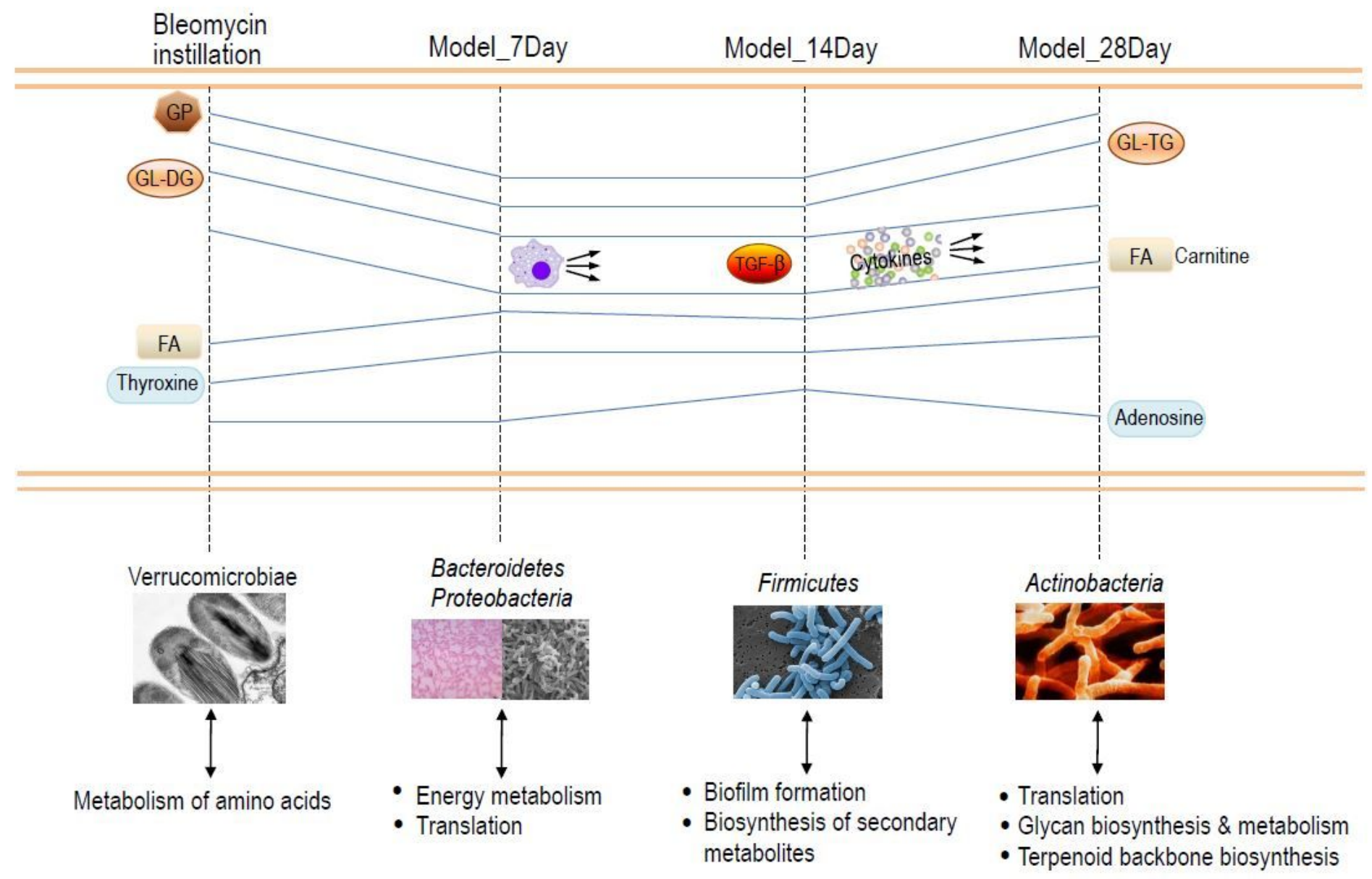

\section{Figure 6}

Graphical summary of the key results of the study. The time dependent changes of major serum metabolites are indicated as trend lines. The representative gut microbiome and the pathways at each time points are shown explicitly.

\section{Supplementary Files}

This is a list of supplementary files associated with this preprint. Click to download.

- TableS1.xIsx

- TableS2.xlsx

- TableS3.xlsx

- Tables4.xIsx 